\title{
Frequency of Adenoviruses, Rotaviruses and Noroviruses Among Diarrhea Samples Collected From Infants of Zabol, Southeastern Iran
}

\author{
Javad Sharifi-Rad $^{1,2}$; Seyedeh Mahsan Hoseini Alfatemi ${ }^{3, *}$; Mehdi Sharifi-Rad ${ }^{4}$; Abdolhossein \\ Miri $^{1,2}$ \\ ${ }^{1}$ Zabol Medicinal Plants Research Center, Zabol University of Medical Sciences, Zabol, IR Iran \\ ${ }^{2}$ Department of Pharmacognosy, Faculty of Pharmacy, Zabol University of Medical Sciences, Zabol, IR Iran \\ ${ }_{3}^{3}$ Pediatric Infections Research Center, Mofid Children's Hospital, Shahid Beheshti University of Medical Sciences, Tehran, IR Iran \\ 4 Zabol Medicinal Plants Research Center, Zabol University of Medical Sciences, Zabol, IR Iran \\ ${ }^{*}$ Corresponding author: Seyedeh Mahsan Hoseini Alfatemi, Pediatric Infections Research Center, Mofid Children's Hospital, Shahid Beheshti University of Medical Sciences, Tehran, \\ IR Iran. Tel:+98-2122439963, E-mail: m.hoseinialfatemi@gmail.com
}

Received: October 15, 2013; Revised: May 27, 2014; Accepted: May 30, 2014

Background: Viruses are one of the major reasons of gastrointestinal disease worldwide, and commonly infect children less than five years of age in developing countries.

Objectives: The current study aimed to determine the frequency of adenoviruses, rotaviruses and noroviruses among diarrhea samples collected from infants of Zabol, south-east of Iran. This study is the first investigation of adenoviruses, rotaviruses and noroviruses among diarrhea samples in Zabol.

Patients and Methods: In this study, eighty-two diarrhea stool samples were collected from infants aged $<1$ to 12 months admitted to the hospital, internal laboratory and central laboratory of Zabol, Iran. All samples were subjected to the rapid immunochromatography assay. Results: The results showed that the frequency of rotaviruses, adenoviruses and noroviruses among infants with diarrhea were $70.20 \%$, $20.30 \%$ and $9.50 \%$, respectively. There were 50 subjects aged one to five months and 2 subjects aged nine to twelve months. The results of geographical distribution showed that the number of infants living in rural and urban areas with these viruses were 50 and 32 , respectively. Rotaviruses were most common in rural and urban infants with 42 and 10 cases, respectively. Regarding the feeding patterns of infants with diarrhea, mixed feeding and breast feeding were found in 51 and 31 cases, respectively.

Conclusions: In conclusion, the results of our study showed that the major viral pathogens that caused infantile diarrhea in Zabol city were rotaviruses followed by adenoviruses and noroviruses. The results of our study can useful for prosperous control of infantile diarrhea.

Keywords:Noroviruses; Rotavirus; Adenoviruses; Infantile Diarrhea

\section{Background}

Diarrhea is the second leading cause of death for children less than five years of age worldwide (1). Despite efforts in the past two decades that have resulted remarkable progress in reducing mortality, diarrhea in children still remains to be a health problem in developing countries. Different types of microbes or parasites can cause diarrhea yet viruses are often the causative agents. Viruses are a cause of gastrointestinal disease worldwide and commonly infect children less than five years of age in developing countries (2). Approximately $10 \%$ of all diarrhea-causing viruses are retroviruses (1).

Rotaviruses are double stranded RNA viruses and belong to the family of Reoviridae that is comprised of seven serogroups (A-G). Rotaviruses are the leading cause that bring about diarrhea hospitalization amongst children worldwide (3). Retroviruses are responsible for deaths of more than two million hospitalized patients and approximately 611000 childhood deaths worldwide annually, mostly from developing countries $(4,5)$. The clinical manifestations of rotaviruses alter from asymptomatic infection to severe and serious, dehydrating diarrhea with vomiting that may be deadly $(6,7)$. Rotaviruses are broadcasted by close contact, particularly through fecaloral route and the respiratory $\operatorname{track}(8,9)$. Widdowson et al. (10) reported that rotavirus infections are the primary cause of serious gastroenteritis in infants around the globe.

Adenoviruses belong to the family Adenoviridae. There are four genera: Mastadenovirus, Aviadenovirus, Atadenovirus and Siadenovirus. Adenoviruses are amongst microorganisms that bringing about severe diarrhea specifically in infants and young children $(11,12)$. Adenovirus infections in children have a wide range of clinical appearances and illness syndromes with various degrees of acuteness. Many of these infections cannot be diagnosed based on clinical criteria.

Noroviruses are from the family Caliciviridae, characterized by a single-stranded, positive-sense, polyadenylated

Copyright (C) 2015, Ahvaz Jundishapur University of Medical Sciences. This is an open-access article distributed under the terms of the Creative Commons Attribution-NonCommercial 4.0 International License (http://creativecommons.org/licenses/by-nc/4.0/) which permits copy and redistribute the material just in noncommercial usages, provided the original work is properly cited. 
RNA genome that contains three open reading frames (ORFs). Noroviruses can lead to gastroenteritis in humans. Human noroviruses have been identified as the most significant cause of nonbacterial, serious gastroenteritis in all age groups. Noroviruses commonly infect children less than five years of age, specifically infants with less than 12 months of age $(13,14)$.

Determining the frequency of these viruses can be useful because it will assist us with disease control and selection of particular treatment and protocols specific for adenoviruses, rotaviruses and noroviruses.

\section{Objectives}

The current study aimed to determine the frequency of adenoviruses, rotaviruses and noroviruses among diarrhea samples collected from infants of Zabol, Southeastern Iran.

\section{Patients and Methods}

\subsection{Sample Collection}

In this study samples were collected from the Hospital, Internal Laboratory and Central Laboratory of Zabol city (Iran). Eighty two diarrhea samples were collected from infants aged between less than one and twelve months. All samples were collected during the winter period (January to March) of the year 2013.

\subsection{Patients Data Collection}

The following demographic data were recorded for each patient; age, geographical region, gender and feeding pattern.

\subsection{Immunochromatography Test}

Immunochromatographic assays are quick strip-based immunoassays whose physical instruction depends on the migration of micro or nanoparticles along a membrane. Results are qualitatively based on Yes/No answer. In this study, for the immunochromatography assay, adenoviruses, rotaviruses and noroviruses were used from cassettes (Biofocus, Korea). Positive results were indicated by two red lines for the control and assay. The stool samples were examined via the immunochromatographic test for adenoviruses, rotaviruses and noroviruses. This assay was based on the use of a homogenous membrane system with colloidal gold particles. The fecal sample was diluted in dilution buffer. A nitro cellulose membrane was sensitized with antibodies directed against adenoviruses, rotaviruses and noroviruses. The assay was performed by methods described by Weitzel et al. (15).

\section{Results}

The results showed that the distribution of rotaviruses, adenoviruses and noroviruses among infants with diarrhea were $70.20 \%, 20.30 \%$ and $9.50 \%$, respectively (Figure 1). The results indicated that there were 22, 50, 8 and 2 infected cases in the age groups of $<1,1-5,5-9$ and 9-12 months (Table 1). The results indicated that the most effected age group was the one to five-month old group with a total number of 50 patients followed by less than one-month group with 22 patients. The geographical information of the infants with diarrhea is indicated in Table 2. These results showed that most cases of viral diarrhea that were caused by rotaviruses ( 34 infant), noroviruses (27 infant) and adenoviruses (21 infant) belonged to rural areas. Overall there were 32 and 50 infants from urban and rural areas, respectively. Regarding the relationship between rotaviruses, adenoviruses, and noroviruses with feeding methods, the results showed that viral diarrheal infections among mixed feeding infants and breast-feeding were 51 and 31, respectively (Table 3). Among breast-feeding infants, rotaviruses, noroviruses, and adenoviruses were found in 14, 12, and 5 cases, respectively. Among mixed feeding infants rotaviruses, noroviruses, adenoviruses were found in 20,15 and 16, respectively.

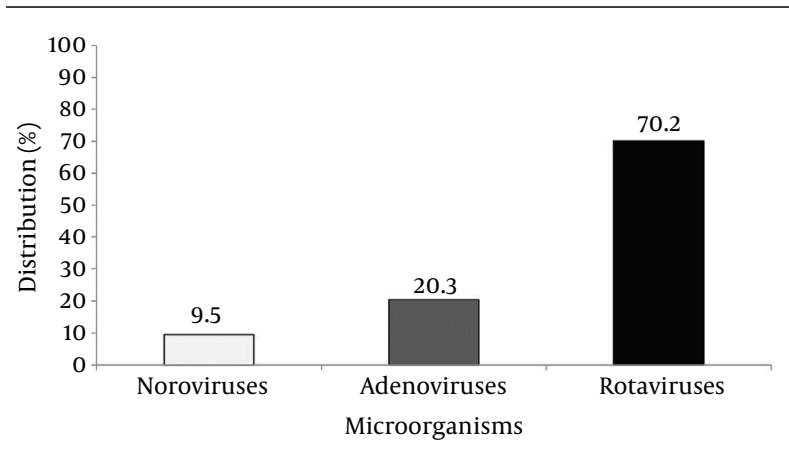

Figure 1. Distribution of Various Viruses Among Infants With Diarrhea

\begin{tabular}{lcccc}
\hline Table 1. Viral Diarrheal Isolates Distribution & & \\
\hline Age Group, Mon & $<\mathbf{1}$ & $\mathbf{1}$ - $\mathbf{5}$ & $\mathbf{5}$ - $\mathbf{9}$ & $\mathbf{9}$ - 12 \\
\hline Rotaviruses & & & & \\
$\quad$ Male & 9 & 6 & 1 & 0 \\
$\quad$ Female & 7 & 8 & 2 & 1 \\
Noroviruses & & & & \\
$\quad$ Male & 3 & 11 & 1 & 0 \\
$\quad$ Female & 0 & 11 & 1 & 0 \\
Adenoviruses & & & & \\
$\quad$ Male & 1 & 5 & 2 & 1 \\
\hline Female & 2 & 9 & 1 & 0 \\
Total & 22 & 50 & 8 & 2 \\
\hline
\end{tabular}


Sharifi Rad J et al.

Table 2. Distribution of Viruses Based on Geographical Distribution of Infants

\begin{tabular}{lcccc}
\hline Type of Virus & Rotaviruses & Noroviruses & Adenoviruses & Total \\
\hline Distribution of Infection & & & & \\
Urban Infant Number & 10 & 16 & 6 & 32 \\
\hline Rural Infant Number & 24 & 11 & 15 & 50 \\
Total & 34 & 27 & 21 & 82 \\
\hline
\end{tabular}

Table 3. Distribution of Viruses Based on the Feeding Patterns of Infants

\begin{tabular}{lcccc}
\hline Type of Virus & Rotaviruses & Noroviruses & Adenoviruses & Total \\
\hline Distribution of Infection Based on Feeding & & & & \\
Breast Feeding Number & 14 & 12 & 5 & 31 \\
\hline Mixed Feeding Number & 20 & 15 & 16 & 51 \\
Total & 34 & 27 & 21 & 82 \\
\hline
\end{tabular}

\section{Discussion}

Our findings were in accordance with that of Elhag et al. (16). They reported that from 99 cases of viral diarrhea, $83.8 \%$ were due to rotaviruses while $16.2 \%$ were due to adenovirus. Motamedifar et al. (17) investigated the frequency of rotavirus and adenovirus gastroenteritis among children in Shiraz, Iran. Rotavirus was identified in 347 patients out of 827 (42\%), adenovirus was detected in 76 (9\%) of the samples and 34 (4\%) patients had rotavirusadenovirus co-infection. Diarrhea was the most common symptom in viral infected patients (17). Sai et al. (18) studied the epidemiology and clinical features of rotavirus and norovirus infection among children in Ji'nan, China. They reported that of the 767 specimens, 263 (34.3\%) were positive for rotavirus and 80 (10.4\%) were positive for norovirus. Tran et al. (19) assayed the prevalence of rotavirus, adenovirus, norovirus and astrovirus infections and co-infections among hospitalized children in Northern France. The results of this study showed that the overall rates of prevalence for rotavirus, norovirus, adenovirus and astrovirus were $21,13,5$ and $1.8 \%$.

Moyo et al. (20) investigated the prevalence of enteropathogenic viruses and molecular characterization of group A rotavirus among children with diarrhea in Dares Salaam Tanzania. They reported that the prevalence of rotavirus, norovirus, adenovirus and astrovirus was $18.1 \%$, $13.7 \%, 2.6 \%$ and $0.4 \%$, respectively. In most cases (62.1\%), viruses were detected in children aged 7-12 months. Similar to our study, the results of all mentioned studies also confirmed the high prevalence of rotavirus among other viruses in children with infection. Kosek et al. (21) reviewed 60 studies on diarrhea morbidity and mortality published from 1990 to 2000 . They concluded that diarrhea accounts for $21 \%$ of all deaths in children younger than five years of age and brings about 2.5 million deaths per year; while diarrhea morbidity has remained without change. Our results regarding feeding patterns were in agreement with those illustrated by Ahmed et al. (22).
They found that vomiting was meaningfully higher in rotaviruses diarrhea as compared to non-rota diarrhea.

The rapid immunochromatography test is easy to carry out at a low cost, and can be an optimum method in place of the latex test for distinguishing adenoviruses, rotaviruses and noroviruses and rapid immunochromatography test (ICGT) exhibited an excellent consent with enzyme immuno assay (EIA) and reverse transcription polymerase chain reaction (RT-PCR) (23). The frequency of illnesses due to rotaviruses is alike in children of both developed and developing countries. However, mortality from this infection is higher in children of developing countries, perhaps due to factors, such as poorer access to hydration therapy and a greater prevalence of undernourishment (23). Lee et al. (23) estimated that 1200 children die from rotavirus-associated illnesses each day, and $82 \%$ of these deaths occur in children of poor countries. The portion of diarrhea deaths associated with rotaviruses can intensify, given that this pathogen is frequently transmitted among people and it is troublesome to control through improving sanitation and hygiene. This assumption is affirmed by information from Mexico, exhibiting that although dying from diarrhea decreased considerably from 1989 to 1995, the decline was less obvious for the winter season in children $<2$ years of age, whose disease had the epidemiological characteristics of rotavirus diarrhea (24).

Dalgic et al. (25) illustrated that the manifestation of this infection usually collaborated with rotaviruses gastroenteritis involving vomiting, diarrhea, fever, lethargy and dehydration, as well as reduced oral intake. Rotaviruses gastroenteritis is possibly linked to high fever and therefore, typical febrile seizures are likely to occur (26). It has been suggested that fever is a noticeable clinical sign of rotaviruses diarrheal infection by the study of Ahmed et al. (22) who found fever in $58.4 \%$ of infants with rotavirus diarrheal infections. The grade of fever was clas- 
sified as “mild" $\left(37.7-38.5^{\circ} \mathrm{C}\right)$, "moderate" $\left(38.6-39.5^{\circ} \mathrm{C}\right)$ and "severe" $\left(>39.6^{\circ} \mathrm{C}\right)$ according to the description of parents and temperature register of the out-patient department and hospitalized patients. This study showed that most infants ( $\leq 12$ months) were infected by viral enteric pathogens and the major viral pathogens causing diarrhea in infants of Zabol city were rotaviruses followed by adenoviruses and noroviruses. This study highlights the need for uninterrupted monitoring of viral pathogens for prosperous treatment and control of diarrhea in infants. We suggest that future studies should be done during one year and results should be interpreted based on each season.

In conclusion, rotaviruses, adenoviruses and noroviruses are significant causes of viral diarrhea in many countries specially developing countries and have led to extensive mortality of children in the past decade. Nonetheless, diarrhea, as a disease with costly treatment, has great impact on the government's financial plan thus determining the causative agents is vital for health policy decision makers. Currently there is a neglect of virus detection assays in clinical laboratories due to lengthy detection procedures, and this has led to unnecessary antibiotic therapy. However, as indicated by the results of this study and previous reports viral screening for rotaviruses, adenoviruses and noroviruses particularly for children less than five years old, appears to be essential.

\section{Acknowledgements}

The authors are very thankful to the Department of Bacteriology and Virology, Shiraz Medical School, Shiraz University of Medical Sciences, Shiraz, Iran for all their cooperation in this study.

\section{Authors' Contributions}

Study idea, design and protocol: Seyedeh Mahsan Hoseini Alfatemi and Javad Sharifi Rad. Immunochromatography assay for quick recognition of adenoviruses, rotaviruses and norovirus: Seyedeh Mahsan Hoseini Alfatemi and Javad Sharifi Rad. Writing of the manuscript: Seyedeh Mahsan Hoseini Alfatemi, Javad Sharifi Rad Mehdi SharifiRad and Abdolhossein Miri.

\section{References}

1. Navaneethan U, Giannella RA. Mechanisms of infectious diarrhea. Nat Clin Pract Gastroenterol Hepatol. 2008;5(11):637-47.

2. Hamedi A, Sadeghian A, Syedi J. Incidence of rotavirus diarrhea in children under 6 years referred to the Pediatric Emergency and Clinic of Ghaem Hospital, Mashhad, Iran. Irani J Pediatr Soc. 2010;2(2):70-4.

3. Parashar UD, Bresee JS, Gentsch JR, Glass RI. Rotavirus. Emerg Infect Dis. 1998;4(4):561-70.

4. Glass RI, Parashar UD, Bresee JS, Turcios R, Fischer TK, Widdowson MA, et al. Rotavirus vaccines: current prospects and future challenges. Lancet. 2006;368(9532):323-32.

5. Parashar UD, Gibson CJ, Bresee JS, Glass RI. Rotavirus and severe childhood diarrhea. Emerg Infect Dis. 2006;12(2):304-6.
6. Fischer TK, Ashley D, Kerin T, Reynolds-Hedmann E, Gentsch J, Widdowson MA, et al. Rotavirus antigenemia in patients with acute gastroenteritis. J Infect Dis. 2005;192(5):913-9.

7. Bernstein DI. Rotavirus overview. Pediatr Infect Dis J. 2009;28(3 Suppl):S50-3.

8. Blutt SE, Conner ME. Rotavirus: to the gut and beyond! Curr Opin Gastroenterol. 2007;23(1):39-43.

9. Ndze VN, Papp H, Achidi EA, Gonsu KH, Laszlo B, Farkas S, et al. One year survey of human rotavirus strains suggests the emergence of genotype G12 in Cameroon. J Med Virol. 2013;85(8):148590.

10. Widdowson MA, Bresee JS, Gentsch JR, Glass RI. Rotavirus disease and its prevention. Curr Opin Gastroenterol. 2005;21(1):26-31.

11. Rocholl C, Gerber K, Daly J, Pavia AT, Byington CL. Adenoviral infections in children: the impact of rapid diagnosis. Pediatrics. 2004;113(1 Pt 1):e51-6.

12. Zlateva KT, Maes P, Rahman M, Van Ranst M. Chromatography paper strip sampling of enteric adenoviruses type 40 and 41 positive stool specimens. Virol J. 2005;2:6.

13. Gutierrez-Escolano AL, Velazquez FR, Escobar-Herrera J, Lopez Saucedo C, Torres J, Estrada-Garcia T. Human caliciviruses detected in Mexican children admitted to hospital during 1998-2000, with severe acute gastroenteritis not due to other enteropathogens. J Med Virol. 2010;82(4):632-7.

14. Gomez-Santiago F, Ribas-Aparicio RM, Garcia-Lozano H. Molecular characterization of human calicivirus associated with acute diarrheal disease in Mexican children. Virol J. 2012;9:54.

15. Weitzel T, Reither K, Mockenhaupt FP, Stark K, Ignatius R, Saad E, et al. Field evaluation of a rota- and adenovirus immunochromatographic assay using stool samples from children with acute diarrhea in Ghana. J Clin Microbiol. 2007;45(8):2695-7.

16. Elhag WI, Saeed HA, Omer el FE, Ali AS. Prevalence of rotavirus and adenovirus associated with diarrhea among displaced communities in Khartoum, Sudan. BMC Infect Dis. 2013;13:209.

17. Motamedifar M, Amini E, Talezadeh Shirazi P. Frequency of rotavirus and adenovirus gastroenteritis among children in shiraz, iran. Iran Red Crescent Med J. 2013;15(8):729-33.

18. Sai L, Sun J, Shao L, Chen S, Liu H, Ma L. Epidemiology and clinical features of rotavirus and norovirus infection among children in Ji'nan, China. Virol J. 2013;10:302.

19. Tran A, Talmud D, Lejeune B, Jovenin N, Renois F, Payan C, et al Prevalence of rotavirus, adenovirus, norovirus, and astrovirus infections and coinfections among hospitalized children in northern France. J Clin Microbiol. 2010;48(5):1943-6.

20. Moyo SJ, Gro N, Kirsti V, Matee MI, Kitundu J, Maselle SY, et al. Prevalence of enteropathogenic viruses and molecular characterization of group A rotavirus among children with diarrhea in Dar es Salaam Tanzania. BMC Public Health. 2007;7:359.

21. Kosek M, Bern C, Guerrant RL. The global burden of diarrhoeal disease, as estimated from studies published between 1992 and 2000. Bull World Health Organ. 2003;81(3):197-204.

22. Ahmed S, Kabir AL, Rahman A, Hussain M, Khatoon S, Hannan A Severity of rotavirus diarrhea in children: one year experience in a children hospital of Bangladesh. Iran J Pediatr. 2009;19(2):108-16.

23. Lee SY, Hong JH, Lee SW, Lee M. [Comparisons of latex agglutination, immunochromatography and enzyme immunoassay methods for the detection of rotavirus antigen]. Korean J Lab Med. 2007;27(6):437-41.

24. Nguyen VM, Nguyen VT, Huynh PL, Dang DT, Nguyen TH, Phan VT, et al. The epidemiology and disease burden of rotavirus in Vietnam: sentinel surveillance at 6 hospitals. J Infect Dis. 2001;183(12):1707-12.

25. Dalgic N, Hasim O, Pullu M, Sancar M, Kafadar I, Yilmaz A. Is Rotavirus Diarrhea a Systemic Viral Infection? Çocuk Enf Derg. 2010;4(2):48-55.

26. Iturriza-Gomara M, Auchterlonie IA, Zaw W, Molyneaux P, Desselberger U, Gray J. Rotavirus gastroenteritis and central nervous system (CNS) infection: characterization of the VP7 and VP4 genes of rotavirus strains isolated from paired fecal and cerebrospinal fluid samples from a child with CNS disease. J Clin Microbiol. 2002;40(12):4797-9. 